\title{
Cationic Copper(I) Complexes as Efficient Pre-catalysts for the Hydrosilylation of Carbonyl Compounds
}

\author{
Silvia Díez-González, Natalie M. Scott and Steven P. Nolan* \\ Department of Chemistry, University of New Orleans, New Orleans, Louisiana 70148
}

snolan@uno.edu

Supporting Information 


\section{General Considerations}

All ketones were used as received. All solid reagents were stored under argon in a glovebox containing less than $1 \mathrm{ppm} \quad \mathrm{O}_{2}$. Tetrakis(acetonitrile)copper(I) hexafluorophosphate and tetrafluoroborate, ${ }^{1} \mathrm{IPr} \cdot \mathrm{HCl}$ and free $\mathrm{IPr}^{2}$ were synthesized according to literature procedures. Solvents were distilled from appropriate drying agents. Flash column chromatography was performed on silica gel 60 (320-400 mesh). ${ }^{1} \mathrm{H}$ NMR and ${ }^{13} \mathrm{C}$ NMR were recorded on a $400 \mathrm{MHz}$ spectrometer at room temperature. Chemical shifts $(\delta)$ are reported with respect to tetramethylsilane as internal standard in ppm. All reported yields are isolated yields and are average of at least two runs.

\section{Synthesis of [(IPr $\left.)_{2} \mathrm{Cu}\right] \mathrm{X}$ Complexes $\left(\mathrm{X}=\mathrm{PF}_{6}\right.$ or $\left.\mathrm{BF}_{4}\right)$}

In an oven-dried vial fitted with a septum screw cap, tetrakis(acetonitrile)copper(I) hexafluoroborate $(0.186 \mathrm{~g}, 0.5 \mathrm{mmol})$ or tetrafluoroborate $(0.157 \mathrm{~g}, 0.5 \mathrm{mmol}) \mathrm{IPr} \cdot \mathrm{HCl}(0.424 \mathrm{~g}, 1 \mathrm{mmol})$ and sodium tert-butoxide (96 mg, $1 \mathrm{mmol}$ ) were loaded inside a glovebox and stirred in dry THF (10 mL) outside the glovebox for 6 hours. After filtering the reaction mixture through a plug of celite (THF), the filtrate was mixed with hexane to form a precipitate. A second filtration led to the isolation of the expected complexes.

Bis[1,3-bis(2,6-diisopropylphenyl)imidazol-2-ylidene]copper hexafluorophosphate (1). From tetrakis(acetonitrile)copper(I) hexafluorophosphate and following the general procedure, $0.474 \mathrm{~g}$ of the title compound were isolated as a pure white solid (96\%). ${ }^{1} \mathrm{H}$ NMR (400 MHz, acetone- $\left.d_{6}\right): \delta=7.54(\mathrm{t}$ containing a singlet at 7.55, $J=7.8 \mathrm{~Hz}, 8 \mathrm{H}, p-\mathrm{CH}+\mathrm{NCH}), 7.25(\mathrm{~d}, J=7.8 \mathrm{~Hz}, 8 \mathrm{H}, m-\mathrm{CH}), 2.42$ (septet, $\left.J=6.8 \mathrm{~Hz}, 8 \mathrm{H},\left(\mathrm{CH}_{3}\right)_{2} \mathrm{CH}\right), 1.04$ (d, $\left.J=6.8 \mathrm{~Hz}, 18 \mathrm{H}, \mathrm{CH}_{3}\right), 0.94\left(\mathrm{~d}, J=6.8 \mathrm{~Hz}, 18 \mathrm{H}, \mathrm{CH}_{3}\right) ;{ }^{13} \mathrm{C}$ NMR (100 MHz; $\left.\mathrm{CDCl}_{3}\right): \delta=178.4(\mathrm{~N}-\mathrm{C}-\mathrm{N}), 145.0$ ( $\mathrm{CH}$ aromatic), 134.6 (C aromatic), 130.6 (C aromatic), $125.2(=\mathrm{CH}-\mathrm{N}), 124.5$ ( $\mathrm{CH}$ aromatic), $28.6\left(\mathrm{CH}\left(\mathrm{CH}_{3}\right)_{2}\right), 24.25\left(\mathrm{CH}_{3}\right), 24.12\left(\mathrm{CH}_{3}\right)$; Elemental analysis calcd for $\mathrm{C}_{54} \mathrm{H}_{72} \mathrm{CuF}_{6} \mathrm{~N}_{4} \mathrm{P}$ (985.69): C, 65.80; H, 7.36; N, 5.68. Found: C, 65.49; H, 7.42; N, 5.99 .

Bis[1,3-bis(2,6-diisopropylphenyl)imidazol-2-ylidene]copper tetrafluoroborate (2). From tetrakis(acetonitrile)copper(I) tetrafluoroborate and following the general procedure, $0.452 \mathrm{~g}$ of the title compound were isolated as a pure white solid (92\%). ${ }^{1} \mathrm{H}$ NMR (400 MHz, acetone- $\left.d_{6}\right): \delta=7.54(\mathrm{t}$ containing a singlet at 7.55, $J=7.5 \mathrm{~Hz}, 8 \mathrm{H}, p-\mathrm{CH}+\mathrm{NCH}), 7.25(\mathrm{~d}, J=7.5 \mathrm{~Hz}, 8 \mathrm{H}, m-\mathrm{CH}), 2.40$ (septet, $\left.J=6.8 \mathrm{~Hz}, 8 \mathrm{H}, \mathrm{CH}\left(\mathrm{CH}_{3}\right)_{2}\right), 1.04$ (d, $\left.J=6.8 \mathrm{~Hz}, 24 \mathrm{H}, \mathrm{CH}_{3}\right), 0.94\left(\mathrm{~d}, J=6.8 \mathrm{~Hz}, 24 \mathrm{H}, \mathrm{CH}_{3}\right) ;{ }^{13} \mathrm{C}$ 
NMR (100 MHz, $\left.\mathrm{CDCl}_{3}\right): \delta=177.4(\mathrm{~N}-\mathrm{C}-\mathrm{N}), 145.0(\mathrm{CH}$ aromatic), 134.7 ( $\mathrm{C}$ aromatic), 130.6 (C aromatic), $125.3(=\mathrm{CH}-\mathrm{N}), 124.5\left(\mathrm{CH}\right.$ aromatic), $28.6\left(\mathrm{CH}\left(\mathrm{CH}_{3}\right)_{2}\right), 24.3\left(\mathrm{CH}_{3}\right), 24.2\left(\mathrm{CH}_{3}\right)$; Elemental analysis calcd for $\mathrm{C}_{54} \mathrm{H}_{72} \mathrm{BCuF}_{4} \mathrm{~N}_{4}$ (927.53): C, 69.93; H, 7.82; N, 6.04. Found: C, 69.57; H, 7.60; N, 5.87 .

\section{General Procedure for the Hydrosilylation of Carbonyl Compounds}

In an oven-dried vial fitted with a septum screw cap, 2 (27 mg, $0.03 \mathrm{mmol}, 3 \mathrm{~mol} \%)$ and sodium tertbutoxide (12 mg, $12 \mathrm{~mol} \%$ ) were charged inside a glove box and stirred in dry THF ( $2 \mathrm{~mL}$ ) at room temperature or at $55{ }^{\circ} \mathrm{C}$ outside of the glove box for 10 minutes before adding triethylsilane $(0.33 \mathrm{~mL}$, 2 mmol, 2 equiv) through the septum using a syringe. After 10 more minutes of stirring, the carbonyl compound ( $1 \mathrm{mmol}$ ) was added. When the starting material was a solid, it was added as a solution in THF. The reaction was monitored by GC, after consumption of the starting material or no further conversion, the reaction mixture was opened to air and filtered through a plug of active charcoal and celite using ethyl acetate as solvent. The organic phase was concentrated in vacuo and the purity of the residue checked on GC and ${ }^{1} \mathrm{H}$ NMR. Flash chromatography was then performed unless crude product was estimated to be greater than $95 \%$ pure.

(Cyclohexyloxy)triethysilane (Table 1, entry 1). Using the general procedure at room temperature, cyclohexanone $(0.100 \mathrm{~mL}, 1 \mathrm{mmol})$ was hydrosilylated by triethylsilane. A colorless oil was obtained as the pure product after concentration of the filtrate $(0.211 \mathrm{~g}$, 98\% yield). Spectroscopic data were consistent with previously reported data for this compound. ${ }^{3}$

(2-Methylcyclohexyloxy)triethylsilane (Table 1, entry 2). Using the general procedure at room temperature, 2-methylcyclohexanone $(0.120 \mathrm{~mL}, 1 \mathrm{mmol})$ was hydrosilylated by triethylsilane. After concentration of the filtrate, a colorless oil was obtained as the pure product in a mixture 85:15 of trans:cis diastereoisomers (0.206 g, 90\% yield). Spectroscopic data were consistent with previously reported data for this compound. ${ }^{4}$

Bicyclo[2.2.1] hept-2-yloxytriethylsilane (Table 1, entry 3). Using the general procedure at room temperature, norcamphor ( $0.110 \mathrm{~g}, 1 \mathrm{mmol})$ was hydrosilylated by triethylsilane. After concentration of the filtrate, a colorless oil was obtained as the pure product in a mixture 97:3 of endo:exo isomers (0.217 g, 96\% yield). Spectroscopic data were consistent with previously reported data for this compound. ${ }^{5}$ 
Trietyl(octan-2-yloxy)silane (Table 1, entry 4). Using the general procedure at room temperature, 2-octanone $(0.156 \mathrm{~mL}, 1 \mathrm{mmol})$ was hydrosilylated by triethylsilane. A colorless oil was obtained as the pure product after concentration of the filtrate $(0.224$ g, 92\% yield). Spectroscopic data were consistent with previously reported data for this compound. ${ }^{6}$

Triethyl(undecan-2-yloxy)silane (Table 1, entry 5). Using the general procedure at room temperature, 2-undecanone $(0.210 \mathrm{~mL}, 1 \mathrm{mmol})$ was hydrosilylated by triethylsilane. A colorless oil was obtained as the pure product after concentration of the filtrate $(0.273 \mathrm{~g}, 97 \%$ yield $)$. Spectroscopic data were consistent with previously reported data for this compound. ${ }^{4}$

(4-Phenylbutan-2-yloxy)triethylsilane (Table 1, entry 6). Using the general procedure at room temperature, benzylacetone $(0.150 \mathrm{~mL}, 1 \mathrm{mmol})$ was hydrosilylated by triethylsilane. A colorless oil was obtained as the pure product after concentration of the filtrate $(0.252 \mathrm{~g}, 95 \%$ yield $)$. Spectroscopic data were consistent with previously reported data for this compound. ${ }^{4}$

(1-Phenylethoxy)triethylsilane (Table 1, entry 7). Using the general procedure at room temperature, acetophenone $(0.117 \mathrm{~mL}, 1 \mathrm{mmol})$ was hydrosilylated by triethylsilane. A colorless oil was obtained as the pure product after concentration of the filtrate $(0.231 \mathrm{~g}, 98 \%$ yield $)$. Spectroscopic data were consistent with previously reported data for this compound. ${ }^{7}$

(1-Phenylpropoxy)triethylsilane (Table 1, entry 8). Using the general procedure at room temperature, propiophenone $(0.133 \mathrm{~mL}, 1 \mathrm{mmol})$ was hydrosilylated by triethylsilane. A colorless oil was obtained as the pure product after concentration of the filtrate $(0.232 \mathrm{~g}, 93 \%$ yield). Spectroscopic data were consistent with previously reported data for this compound. ${ }^{5}$

[1-(Naphthalen-1-yl)ethoxy]triethylsilane (Table 1, entry 9). Using the general procedure at room temperature, 1 '-acetonaphtone $(0.154 \mathrm{~mL}, 1 \mathrm{mmol})$ was hydrosilylated by triethylsilane. A colorless oil was obtained as the pure product after concentration of the filtrate $(0.269 \mathrm{~g}, 94 \%$ yield). Spectroscopic data were consistent with previously reported data for this compound. ${ }^{5}$

Triethyl(hex-5-en-2-yloxy)silane (Tabe 1, entry 10). Using the general procedure at room temperature, hex-5-en-2-one $(0.117 \mathrm{~mL}, 1 \mathrm{mmol})$ was hydrosilylated by triethylsilane. A colorless oil was obtained as the pure product after concentration of the filtrate $(0.220 \mathrm{~g}, 98 \%$ yield $)$. Spectroscopic data were consistent with previously reported data for this compound. ${ }^{8}$

(1-Isopropyl-2-methylpropoxy)triethylsilane (Table 2, entry 1). Using the general procedure at 55 ${ }^{\circ} \mathrm{C}$, 2,4-dimethylpentanone $(0.140 \mathrm{~mL}, 1 \mathrm{mmol})$ was hydrosilylated by triethylsilane. The residue was purified by flash chromatography on silica gel (pentane) to afford $0.214 \mathrm{~g}$ (93\% yield) of the title compound as a colorless oil. Spectroscopic data were consistent with previously reported data for this compound. ${ }^{9}$ 
(2,6-Dimethylcyclohexyloxy)triethylsilane (Table 2, entry 2). Using the general procedure at 55 ${ }^{\circ} \mathrm{C}$ with $19.3 \mathrm{mg}$ (20 mol \%) of $\mathrm{NaO}^{t} \mathrm{Bu}, 2,6$-dimethylcyclohexanone $(0.140 \mathrm{~mL}, 1 \mathrm{mmol})$ was hydrosilylated by triethylsilane. The crude product was purified by flash chromatography on silica gel (pentane) to afford two diastereoisomers of the title compound ( $0.052 \mathrm{~g}$ of the meso trans/trans isomer and $0.174 \mathrm{~g}$ of the meso cis/cis, $93 \%$ yield) ${ }^{10}$ as colorless oils. Spectroscopic data were consistent with previously reported data for this compound. ${ }^{11}$

(Dicyclopropylmethoxy)triethylsilane (Table 2, entry 3). Using the general procedure at $55{ }^{\circ} \mathrm{C}$, dicyclopropyl ketone $(0.114 \mathrm{~mL}, 1 \mathrm{mmol})$ was hydrosilylated by triethylsilane. The crude product was purified by flash chromatography on silica gel (pentane) to afford $0.211 \mathrm{~g}$ the title compound as a colorless oil (94\% yield). Spectroscopic data were consistent with previously reported data for this compound. $^{5}$

(Dicyclohexylmethoxy)triethylsilane (Table 2, entry 4). Using the general procedure at $55{ }^{\circ} \mathrm{C}$ with $0.50 \mathrm{~mL}(3 \mathrm{mmol})$ of triethylsilane, dicyclohexyl ketone $(0.200 \mathrm{~mL}, 1 \mathrm{mmol})$ was hydrosilylated by triethylsilane. The crude product was purified by flash chromatography on silica gel (pentane) to afford $0.304 \mathrm{~g}$ the title compound as a colorless oil (98\% yield). Spectroscopic data were consistent with previously reported data for this compound. ${ }^{11}$

Triethyl-(1-o-tolylethoxy)silane (Table 2, entry 5). Using the general procedure at $55{ }^{\circ} \mathrm{C}, 2^{\prime}-$ methylacetophenone $(0.175 \mathrm{~mL}, 1 \mathrm{mmol})$ was hydrosilylated by triethylsilane. The crude product was purified by flash chromatography on silica gel (pentane:Et ${ }_{2} \mathrm{O}, 95: 5$ ) to afford $0.241 \mathrm{~g}$ (96\% yield) of the title compound as a colorless oil. Spectroscopic data were consistent with previously reported data for this compound. ${ }^{11}$

(Diphenylmethoxy)triethylsilane (Table 2, Entry 6). Using the general procedure at $55{ }^{\circ} \mathrm{C}$, benzophenone $(0.189 \mathrm{~g}, 1 \mathrm{mmol})$ was hydrosilylated by triethylsilane. The crude product was purified by flash chromatography on silica gel (pentane:Et ${ }_{2} \mathrm{O}, 95: 5$ ) to afford $0.276 \mathrm{~g}$ (93\% yield) of the title compound as a colorless oil. Spectroscopic data were consistent with previously reported data for this compound. $^{11}$

(2,2-Dimethyl-1-phenylpropoxy)triethylsilane (Table 2, entry 7). Using the general procedure at $55{ }^{\circ} \mathrm{C}$, 2,2-dimethylpropiophenone $(0.168 \mathrm{~mL}, 1.0 \mathrm{mmol})$ was hydrosilylated by triethylsilane. The crude product was purified by flash chromatography on silica gel (pentane) to afford $0.274 \mathrm{~g}$ (99\% yield) of the title compound as a colorless oil. Spectroscopic data were consistent with previously reported data for this compound. ${ }^{11}$

(4-Methylbenzyloxy)triethylsilane (Table 3, entry 1). Using the general procedure at room temperature, $p$-tolulaldehyde $(0.118 \mathrm{~mL}, 1 \mathrm{mmol})$ was hydrosilylated by triethylsilane. The crude 
product was purified by flash chromatography on silica gel (pentane:Et ${ }_{2} \mathrm{O}$, 98:2) to afford $0.218 \mathrm{~g}(92 \%$ yield) of the title compound as a colorless oil. ${ }^{1} \mathrm{H}$ NMR (400 $\left.\mathrm{MHz}, \mathrm{CDCl}_{3}\right): \delta=7.24(\mathrm{~d}, J=8.1 \mathrm{~Hz}$, 2H, $H$ aromatic), 7.15 (d, $J=8.1 \mathrm{~Hz}, H$ aromatic), 4.71 (s, 2H, OCH $), 2.35$ (s, 3H, $\left.\mathrm{CH}_{3}\right), 0.99$ (t, $J=$ $\left.8.0 \mathrm{~Hz}, 9 \mathrm{H}, \mathrm{SiCH}_{2} \mathrm{CH}_{3}\right), 0.66$ (q, $\left.J=8.0 \mathrm{~Hz}, 6 \mathrm{H}, \mathrm{SiCH}_{2} \mathrm{CH}_{3}\right) ;{ }^{13} \mathrm{C} \mathrm{NMR}\left(100 \mathrm{MHz}, \mathrm{CDCl}_{3}\right): \delta=138.2$ (C aromatic), 136.3 (C aromatic), 128.7 ( $\mathrm{CH}$ aromatic), $126.2\left(\mathrm{CH}\right.$ aromatic), $64.5\left(\mathrm{OCH}_{2}\right), 20.9\left(\mathrm{CH}_{3}\right)$, $6.6\left(\mathrm{SiCH}_{2} \mathrm{CH}_{3}\right), 4.5\left(\mathrm{SiCH}_{2} \mathrm{CH}_{3}\right)$; Elemental analysis calcd for $\mathrm{C}_{14} \mathrm{H}_{24} \mathrm{OSi}$ (236.43): C, 71.12; $\mathrm{H}$, 10.23. Found: C, 71.33; H, 10.02.

(2-Methylbenzyloxy)triethylsilane (Table 3, entry 2). Using the general procedure at room temperature, o-tolulaldehyde $(0.117 \mathrm{~mL}, 1 \mathrm{mmol})$ was hydrosilylated by triethylsilane. The crude product was purified by flash chromatography on silica gel (pentane:Et ${ }_{2} \mathrm{O}$, 95:5) to afford $0.221 \mathrm{~g}(94 \%$ yield) of the title compound as a colorless oil. Spectroscopic data were consistent with previously reported data for this compound. ${ }^{12}$

(4-Fluorobenzyloxy)triethylsilane (Table 3, entry 3). Using the general procedure at room temperature, $p$-fluorobenzaldehyde $(0.100 \mathrm{~mL}, 1 \mathrm{mmol})$ was hydrosilylated by triethylsilane. The crude product was purified by flash chromatography on silica gel (pentane) to afford $0.231 \mathrm{~g}$ (96\% yield) of the title compound as a colorless oil. Spectroscopic data were consistent with previously reported data for this compound. ${ }^{12}$

(4-Chlorobenzyloxy)triethylsilane (Table 3, entry 4). Using the general procedure at room temperature, $p$-chlorobenzaldehyde $(0.140 \mathrm{~mL}, 1 \mathrm{mmol})$ was hydrosilylated by triethylsilane. The crude product was purified by flash chromatography on silica gel (pentane:Et ${ }_{2} \mathrm{O}, 95: 5$ ) to afford 0.234 g (91\% yield) of the title compound as a colorless oil. ${ }^{1} \mathrm{H}$ NMR $\left(400 \mathrm{MHz}, \mathrm{CDCl}_{3}\right): \delta=7.32-7.18(\mathrm{~m}$, 4H, $H$ aromatic), 4.70 (s, 2H, $\mathrm{OCH}_{2}$ ), 0.98 (t, $\left.J=8.0 \mathrm{~Hz}, 9 \mathrm{H}, \mathrm{SiCH}_{2} \mathrm{CH}_{3}\right), 0.65$ (q, $J=8.0 \mathrm{~Hz}, 6 \mathrm{H}$, $\left.\mathrm{SiCH}_{2} \mathrm{CH}_{3}\right) ;{ }^{13} \mathrm{C}$ NMR (100 MHz, $\left.\mathrm{CDCl}_{3}\right): \delta=139.8$ ( $\mathrm{C}$ aromatic), 132.5 ( $\mathrm{C}$ aromatic), $128.3(\mathrm{CH}$ aromatic), $127.4\left(\mathrm{CH}\right.$ aromatic), $65.0\left(\mathrm{OCH}_{2}\right), 6.7\left(\mathrm{SiCH}_{2} \mathrm{CH}_{3}\right), 4.4\left(\mathrm{SiCH}_{2} \mathrm{CH}_{3}\right)$; Elemental analysis calcd for $\mathrm{C}_{13} \mathrm{H}_{21} \mathrm{ClOSi}$ (256.84): C, 60.79; H, 8.24. Found: C, 60.83; H, 8.31.

(2-Chlorobenzyloxy)triethylsilane (Table 3, entry 5). Using the general procedure at room temperature, o-chlorobenzaldehyde $(0.112 \mathrm{~mL}, 1 \mathrm{mmol})$ was hydrosilylated by triethylsilane. The crude product was purified by flash chromatography on silica gel (pentane:Et ${ }_{2} \mathrm{O}, 98: 2$ ) to afford 0.242 g (94\% yield) of the title compound as a colorless oil. ${ }^{1} \mathrm{H}$ NMR $\left(400 \mathrm{MHz}, \mathrm{CDCl}_{3}\right): \delta=7.58$ (d, $J=7.5$ $\mathrm{Hz}, 1 \mathrm{H}, H$ aromatic), 7.32-7.21 (m, 2H, $H$ aromatic), 7.21-7.14 (m, 1H, $H$ aromatic), 4.80 (s, 2H, $\mathrm{OCH}_{2}$ ), 0.99 (t, $\left.J=8.1 \mathrm{~Hz}, 9 \mathrm{H}, \mathrm{SiCH}_{2} \mathrm{CH}_{3}\right), 0.69$ (q, $\left.J=8.1 \mathrm{~Hz}, 6 \mathrm{H}, \mathrm{SiCH}_{2} \mathrm{CH}_{3}\right) ;{ }^{13} \mathrm{C} \mathrm{NMR}(100 \mathrm{MHz}$, $\left.\mathrm{CDCl}_{3}\right): \delta=138.8$ ( $C$ aromatic), 131.3 ( $C$ aromatic), 128.7 ( $C H$ aromatic), 127.8 ( $C H$ aromatic), 127.4 
(CH aromatic), 126.6 ( $\mathrm{CH}$ aromatic), $61.9\left(\mathrm{OCH}_{2}\right), 6.7\left(\mathrm{SiCH}_{2} \mathrm{CH}_{3}\right), 4.4\left(\mathrm{SiCH}_{2} \mathrm{CH}_{3}\right)$; Elemental analysis calcd for $\mathrm{C}_{13} \mathrm{H}_{21}$ ClOSi (256.84): C, 60.79; H, 8.24. Found: C, 60.92; H, 8.40.

(4-Bromobenzyloxy)triethylsilane (Table 3, entry 6). ${ }^{12}$ Using the general procedure at room temperature, $p$-bromobenzaldehyde $(0.185 \mathrm{~g}, 1 \mathrm{mmol})$ was hydrosilylated by triethylsilane. The crude product was purified by flash chromatography on silica gel (pentane: $\mathrm{Et}_{2} \mathrm{O}$, 98:2) to afford $0.281 \mathrm{~g}(93 \%$ yield) of the title compound as a colorless oil. Spectroscopic data were consistent with previously reported data for this compound. Elemental analysis calcd for $\mathrm{C}_{13} \mathrm{H}_{21} \mathrm{BrOSi}$ (301.29): C, 51.82; $\mathrm{H}$, 7.03. Found: C, 52.03; H, 7.28.

(4-Methoxybenzyloxy)triethylsilane (Table 3, entry 7). Using the general procedure at room temperature, $p$-anisaldehyde $(0.120 \mathrm{~mL}, 1 \mathrm{mmol})$ was hydrosilylated by triethylsilane. The crude product was purified by flash chromatography on silica gel (pentane: $\mathrm{Et}_{2} \mathrm{O}$, 98:2) to afford $0.225 \mathrm{~g}(89 \%$ yield) of the title compound as a colorless oil. Spectroscopic data were consistent with previously reported data for this compound. ${ }^{13}$

(Cyclohexylmethoxy)triethylsilane (Table 3, entry 8). Using the general procedure at room temperature with $0.50 \mathrm{~mL}$ (3 mmol, 3 equiv) of triethylsilane, cyclohexyl carbaldehyde $(0.120 \mathrm{~mL}, 1$ mmol) was hydrosilylated by triethylsilane. The crude product was purified by flash chromatography on silica gel (pentane: $\mathrm{Et}_{2} \mathrm{O}, 98: 2$ ) to afford $0.223 \mathrm{~g}$ (70\% yield) of the title compound as a colorless oil. ${ }^{1} \mathrm{H}$ NMR (400 MHz, $\mathrm{CDCl}_{3}$ ): $\delta=3.39$ (d, $J=6.4 \mathrm{~Hz}, 2 \mathrm{H}, \mathrm{OCH}_{2}$ ), 1.87-1.59 (m, 6H, cyclohexyl), 1.57-1.38 (m, 2H, cyclohexyl), 1.36-1.04 (m, 5H, cyclohexyl), 0.97 (t, $J=8.1 \mathrm{~Hz}, 9 \mathrm{H}, \mathrm{SiCH}_{2} \mathrm{CH}_{3}$ ), 0.60 (q, $\left.J=8.1 \mathrm{~Hz}, 6 \mathrm{H}, \mathrm{SiCH}_{2} \mathrm{CH}_{3}\right) ;{ }^{13} \mathrm{C} \mathrm{NMR}\left(100 \mathrm{MHz}, \mathrm{CDCl}_{3}\right): \delta=68.6\left(\mathrm{OCH}_{2}\right), 40.5(\mathrm{CH}), 29.8$ $\left(\mathrm{CH}_{2}\right), 26.8\left(\mathrm{CH}_{2}\right), 25.9\left(\mathrm{CH}_{2}\right), 6.8\left(\mathrm{SiCH}_{2} \mathrm{CH}_{3}\right), 4.4\left(\mathrm{SiCH}_{2} \mathrm{CH}_{3}\right)$; Elemental analysis calcd for $\mathrm{C}_{13} \mathrm{H}_{28} \mathrm{OSi}$ (228.45): C, 68.35; H, 12.35. Found: C, 68.44; H, 12.51.

(2,6-Dimethylhept-5-enyloxy)triethylsilane (Table 3, entry 9). Using the general procedure at room temperature with $0.50 \mathrm{~mL}$ ( $3 \mathrm{mmol}, 3$ equiv) of triethylsilane, 2,6-dimethylhept-5-enal (0.160 $\mathrm{mL}, 1 \mathrm{mmol}$ ) was hydrosilylated by triethylsilane. The crude product was purified by flash chromatography on silica gel (pentane:Et $\left.{ }_{2} \mathrm{O}, 98: 2\right)$ to afford $0.183 \mathrm{~g}$ (71\% yield) of the title compound as a colorless oil. ${ }^{1} \mathrm{H}$ NMR $\left(400 \mathrm{MHz} \mathrm{CDCl}_{3}\right): \delta=5.13$ (broad t, $\left.J=7.5 \mathrm{~Hz}, 1 \mathrm{H}, \mathrm{C}=\mathrm{CH}\right), 3.47$ (dd, $J=$ 5.8, $9.9 \mathrm{~Hz}, 1 \mathrm{H}, \mathrm{OCH}_{2}$ ), 3.36 (dd, $\left.J=6.8,9.9 \mathrm{~Hz}, 1 \mathrm{H}, \mathrm{OCH}_{2}\right), 2.10-1.88\left(\mathrm{~m}, 2 \mathrm{H}, \mathrm{C}=\mathrm{CH}-\mathrm{CH}_{2}\right), 1.69(\mathrm{~s}$, $3 \mathrm{H}, \mathrm{CH}_{3}-\mathrm{C}=$ ), 1.65-1.53 (m containing a singlet at 1.61, 4H, $\left.\mathrm{CH}_{3}-\mathrm{CH}+\mathrm{CH}_{3}-\mathrm{C}=\right)$, 1.49-1.37 (m, $1 \mathrm{H}$, $\mathrm{CH}-\mathrm{CH}_{2}$ ), 1.15-1.02 (m, 1H, CH-CH $), 0.97$ (t, 9H, $\left.J=7.9 \mathrm{~Hz}, \mathrm{SiCH}_{2} \mathrm{CH}_{3}\right), 0.60$ (q, $J=7.9 \mathrm{~Hz}, 6 \mathrm{H}$, $\left.\mathrm{SiCH}_{2} \mathrm{CH}_{3}\right) ;{ }^{13} \mathrm{C}$ NMR (100 MHz, $\left.\mathrm{CDCl}_{3}\right): \delta=131.1(\mathrm{C}=\mathrm{CH}), 124.9(\mathrm{C}=\mathrm{CH}), 68.1\left(\mathrm{OCH}_{2}\right), 35.4$ $\left(=\mathrm{CH}-\mathrm{CH}_{2}\right), 33.4\left(\mathrm{CH}-\mathrm{CH}_{2}\right), 25.7\left(=\mathrm{C}-\mathrm{CH}_{3}\right), 25.5\left(=\mathrm{C}-\mathrm{CH}_{3}\right), 17.6\left(\mathrm{CH}_{2}-\mathrm{CH}\right), 16.7\left(\mathrm{CH}_{3}\right), 6.8$ 
( $\left.\mathrm{SiCH}_{2} \mathrm{CH}_{3}\right)$, $4.4\left(\mathrm{SiCH}_{2} \mathrm{CH}_{3}\right)$; Elemental analysis calcd for $\mathrm{C}_{15} \mathrm{H}_{32} \mathrm{OSi}$ (256.50): C, 70.24; H, 12.57 . Found: C, 70.50; H, 12.74 .

Triethyl(phenethyloxy)silane (Scheme 3). Using the general procedure at $55{ }^{\circ} \mathrm{C}$ with $0.50 \mathrm{~mL}(3$ $\mathrm{mmol})$ of triethylsilane, ethyl phenylacetate $(0.160 \mathrm{~mL}, 1 \mathrm{mmol})$ was hydrosilylated by triethylsilane. The crude product was purified by flash chromatography on silica gel (pentane:Et $2 \mathrm{O}, 98: 2$ ) to afford $0.154 \mathrm{~g}$ the title compound as a colorless oil (65\% yield). ${ }^{1} \mathrm{H}$ NMR (400 $\left.\mathrm{MHz}, \mathrm{CDCl}_{3}\right): \delta=7.26-7.18$ (m, 2H, $H$ aromatic), 7.18-7.08 (m, 3H, 2H, $H$ aromatic), 3.73 (t, $\left.J=7.6 \mathrm{~Hz}, 2 \mathrm{H}, \mathrm{OCH}_{2}\right), 2.77$ (t, $J=$ 7.6 Hz, 2H, Ph-CH $\mathrm{CH}_{2}$, 0.86 (t, 9H, $J=8.0 \mathrm{~Hz}, \mathrm{SiCH}_{2} \mathrm{CH}_{3}$ ), 0.50 (q, $J=8.0 \mathrm{~Hz}, 6 \mathrm{H}, \mathrm{SiCH}_{2} \mathrm{CH}_{3}$ ); ${ }^{13} \mathrm{C}$ NMR (100 MHz, $\left.\mathrm{CDCl}_{3}\right): \delta=139.1(\mathrm{C}), 129.1$ ( $\mathrm{CH}$ aromatic), 128.2 ( $\mathrm{CH}$ aromatic), 126.1 ( $\mathrm{CH}$ aromatic), $64.2\left(\mathrm{OCH}_{2}\right), 39.7\left(\mathrm{Ph}-\mathrm{CH}_{2}\right), 6.7\left(\mathrm{SiCH}_{2} \mathrm{CH}_{3}\right), 4.4\left(\mathrm{SiCH}_{2} \mathrm{CH}_{3}\right)$; Elemental analysis calcd for $\mathrm{C}_{14} \mathrm{H}_{24} \mathrm{OSi}$ (236.43): C, 71.12; H, 10.23. Found: C, 71.27; H, 10.44.

\section{Mechanistic studies}

In an oven-dried vial fitted with a septum screw cap, $\left[\mathrm{Cu}\left(\mathrm{CH}_{3} \mathrm{CN}\right)_{4}\right] \mathrm{BF}_{4}(9 \mathrm{mg}, 0.03 \mathrm{mmol}, 3 \mathrm{~mol}$ \%), sodium tert-butoxide (11.5 mg, 12 mol\%) and IPr (12 mg, 3 mol\% or 23 mg, 6 mol\% or 35 mg, 9 mol\%) were loaded inside a glove box and stirred in dry THF (2 mL) at room temperature outside of the glove box for 40 minutes $^{14}$ before adding triethylsilane $(0.33 \mathrm{~mL}, 2 \mathrm{mmol}, 2$ equiv) through the septum using a syringe. After 10 minutes of stirring, cyclohexanone $(0.100 \mathrm{~mL}, 1 \mathrm{mmol})$ was added. The reactions were monitored by GC, the different reaction rates are presented in Figure 1. The formation of the expected silyl ether was confirmed by ${ }^{1} \mathrm{H}$ NMR. 


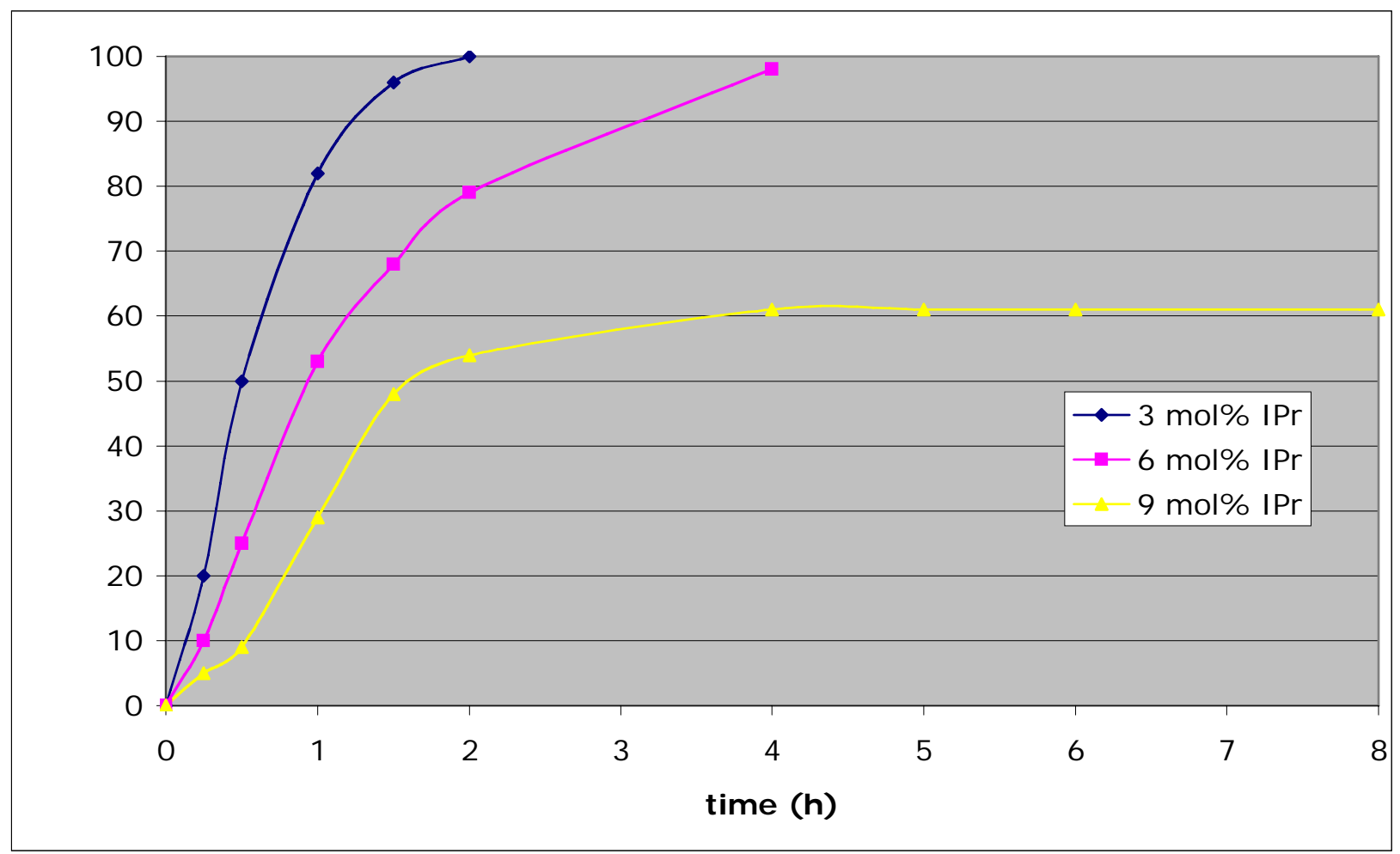

Figure 1. Influence of ligand loading in the hydrosilylation of cyclohexanone

\section{Crystallographic Data}

Crystallographic information files (CIF) of complexes 1 and 2 have been deposited with the CCDC, 12 Union Road, Cambridge, CB2 1EZ, U.K. [fax: (+44) 1223 336033, email: deposit@ccdc.cam.ac.uk] and can be obtained on request free of charge, by quoting the publication citation and deposition number 279243 (1) and 279244 (2).

\section{References and Notes}

[1] Kubas, G. J. Inorg. Synth. 1979, 19, 90-92.

[2] For synthesis of NHC see: a) Huang, J.; Nolan, S. P. J. Am. Chem. Soc. 1999, 120, 9889-9890. b) Arduengo, A. J. III; Calabrese, J. C.; Davidson, F.; Rasika Dias, H. V.; Goerlich, J. R.; Krafczyk, R.; Marshall, W. J.; Tamm, M.; Schulmutzler, R. Helv. Chim. Acta 1999, 82, 2348-2364 and references herein. c) Trnka, T. M.; Grubbs, R. H. Acc. Chem. Res. 2001, 34, 18-29 and references herein. [3] Onaka, M.; Higuchi, K.; Nanami, H.; Izumi, Y. Bull. Chem. Soc. Jpn. 1993, 66, 2638-2645. [4] Lipshutz, B. H.; Caires, C. C.; Kunipers, P.; Chrisman, W. Org. Lett. 2003, 5, 3085-3088. 
[5] Kaur, H.; Zinn, F. K.; Stevens, E. D.; Nolan, S. P. Organometallics 2004, 23, 1157-1160.

[6] Doyle, M. P.; High, K. G.; Bagheri, V.; Pieters, R. J.; Lewis, P. J.; Pearson, M. M. J. Org. Chem. 1990, 55, 6082-6086.

[7] Field, L. D.; Messerle, B. A.; Rehr, M.; Soler, L. P.; Hambley, T. W. Organometallics 2003, 22, 2387-2395.

[8] Dioumaev, V. K.; Bullock, R. M. Nature 2003, 424, 530-532.

[9] Barden, J.; Fleming, J. Chem. Commun. 2001, 22, 2366-2367.

[10] In the second fraction, two diastereoisomers were present but only the major one, the meso cis/cis, could be fully characterized. The presence of the cis/trans isomer as minor product was evidenced by the presence of a doublet of doublets at $3.29 \mathrm{ppm}(J=7.6,4.0 \mathrm{~Hz})$ in the ${ }^{1} \mathrm{H}$ NMR spectrum.

[11] Díez-González, S.; Kaur, H.; Kauer, F. Z.; Stevens, E. D.; Nolan, S. P. J. Org. Chem. 2005, 70, 4784-4796.

[12] Gevorgyan, V.; Rubin, M.; Lui, J.-X.; Yamamoto, Y. J. Org. Chem. 2001, 66, 1672-1675.

[13] Kennedy-Smith, J. J.; Nolin, K. A.; Gunterman, H. P.; Toste, F. D. J. Am. Chem. Soc. 2003, 125, 4056-4057.

[14] When this mixture was stirred for only ten minutes different reaction rates were observed and the best results were obtained for $9 \mathrm{~mol} \%$ of IPr. This may be explained by a slow formation of the active mono-carbene complex that should be favored in the presence of an excess of ligand. 